Observat i on of opt i cal emi ssi on from hi gh refractive i ndex wavegui de excited by travel ing el ectron beam

\begin{tabular}{|l|l|}
\hline 著者 & $\begin{array}{l}\text { Kuwamur a Yuj i, Yamada M nor u, Okandt o Ryui chi , } \\
\text { Kanai Takeshi, Far es Hesham }\end{array}$ \\
\hline $\begin{array}{l}\text { j our nal or } \\
\text { publ i cat i on t i t l e }\end{array}$ & $\begin{array}{l}2008 \text { Conf er ence on Quant um El ect r oni cs and } \\
\text { Laser Sci ence Conf er ence on Laser s and } \\
\text { El ect r o- Opt i cs, CLEO/QELS }\end{array}$ \\
\hline vol ume & 2008 \\
\hline page r ange & 4551231 \\
\hline year & 2008 01-01 \\
\hline URL & ht t p: //hdl . handl e. net /2297/11860 \\
\hline
\end{tabular}




\title{
Observation of optical emission from high refractive index waveguide excited by traveling electron beam
}

\author{
Yuji Kuwamura, Minoru Yamada, Ryuichi Okamoto, Takeshi Kanai and Hesham Fares \\ Graduate School of Natural Science and Technology, University of Kanazawa, Kakuma-machi, Kanazawa, Ishikawa 920-1192, Japan \\ kuwamura@t.kanazawa-u.ac.jp
}

\begin{abstract}
A new scheme for optical emission using a high refractive index waveguide and the traveling electron beam in vacuum was demonstrated. Optical emission around wavelength of $1.5 \mu \mathrm{m}$ was observed for electron acceleration voltage of $40 \mathrm{KV}$.

(C) 2008 Optical Society of America

OCIS codes: (250.4480) Optoelectronics; (350.5610) Other areas of optics
\end{abstract}

\section{Introduction}

Technology of opto-electronics have been successfully developed with invention of lasers. However, a lacking feature of conventional lasers is not having uni-directional property for optical amplification. We had theoretically proposed a scheme [1,2] to realize the unidirectional optical amplification, where a forward propagating optical light can be amplified but another field component propagating in backward direction is never amplified. This new type of amplifier consists of a high refractive index waveguide and an electron gun in the vacuum environment. This amplifier also should work as an optical emitter by same principle with the amplifier. Here we report experimental observation of optical emission to ensure this new scheme.

\section{Principle of optical emission}

Configuration of this experiment is illustrated in Fig.1. A slab waveguide whose core has high refractive index is set in a vacuum chamber with an electron gun. Electron beam is emitted from the electron gun with accelerated voltage $V$ and travels along surface of the optical waveguide. The waveguide is designed to propagate an optical guided mode in the direction of the traveling electron and a part of the optical field penetrates into vacuum space. The guided mode should have an electric component (polarization) along propagating direction. That is a TM (transverse magnetic) mode in the slab waveguide. The electron beam can interact with the optical guided mode through penetrated portion into the vacuum space.

When the group velocity $v_{e}$ of the traveling electron becomes slightly faster than the phase velocity $v_{\text {opt }}$, optical emission is generated. This type of optical emission is belong regime of Cerenkov radiation. The optical emission is one type of spontaneous emission because there is no artificially incident optical field , but we call here to be guided spontaneous emission to distinguish from conventional spontaneous emission which emits for all spatial directions with all polarizations.

The phase velocity $v_{\text {opt }}$ is decided not only with refractive indices of material but also with thickness of core layer because the optical field distributes into two cladding regions upper and down sides. Then the optical velocity $v_{\text {opt }}$ and the acceleration voltage $V$ to keep condition of $v_{e}=v_{\text {opt }}$ change with wavelength of the emitted 
light. Theoretically calculated dispersion relations between wavelength of the optical emission and the acceleration voltage in this experiment are shown in Fig.2 with solid lines. Dots are experimentally obtained data as shown in the next section.

\section{Experimental observation}

An electron gun for RHEED measurement was diverted for this experiment. The electron accelerating voltage was in the range of $30-50 \mathrm{KV}$ and the electron current was around $50 \mu \mathrm{A}$. The optical waveguide was constructed from a SOI substrate for LSI circuits. The top Si layer was used as the core layer having refractive index of $n_{1}=3.485$ and thickness of $d=0.32 \mu \mathrm{m}$ The connecting . $\mathrm{SiO}_{2}$ layer was used as lower side cladding layer having refractive index of $n_{2}=1.444$ with thickness of $1 \mu \mathrm{m}$. The field did not spread in to the bottom $\mathrm{Si}$ layers. The effective refractive index $n_{\text {eff }}=\mathrm{C} / v_{\text {opt }}$ for the TM fundamental mode in the waveguide is $2.5-2.9$ in the wavelength range of $1.2-1.6 \mu \mathrm{m}$.

There was no incident light used in the previous our experiment [3].

The optical light was emitted from edge of the waveguide and was taken through a viewport of the vacuum chamber to outside space and detected after passing a polarization analyzer and monochromator.

Examples of measured spectrum are shown in Fig.3 for the electron accelerating voltage of $40 \mathrm{KV}$. Closed circles in this figure are data of the TM mode which has an electric field component along traveling direction. Open circles are data of the TE (transverse electric) mode which has no electric component along traveling direction. The optical emission was observed for the TM mode, but was not for the TE mode.

Variation of the emitting spectrum for different electron accelerating voltage $V$ are shown in Fig.4. The peak wavelength of the emission spectrum shifted greatly from $1.28 \mu \mathrm{m}$ to $1.6 \mu \mathrm{m}$ with increase of the acceleration voltage from $32 \mathrm{KV}$ to $42 \mathrm{KV}$. The dots shown in Fig.2 were as traces of the peak wavelength of the emission with the acceleration voltage.

Since Si crystal is transparent for longer wavelength than $1.2 \mu \mathrm{m}$, these emission are never conventional optical emission by material itself.

\section{Conclusions}

We theoretically predicted a new scheme of optical emission using traveling electron beam and slowed down optical light. Optical emission based on proposed scheme was experimentally observed and detailed conditions to get the emission were determined.

\section{References}

[1] M. Yamada, “Theoretical proposal for a unidirectional optical amplifier, ” IEEE J. of Quantum Electron 35, 147-152 (1999).

[2] M. Asada and M. Yamada, "Theoretical analysis of interaction between electron beam and electromagnetic wave for unidirectional optical amplifier,” J. Appl. Phys. 95, 5123-5130(2004).

[3] Y. Kuwamura, M.Yamada, Y. Ichino, I. Fukamura and T. Sekikawa, “Observation of optical amplification excited by traveling electron beam,” Jpn. J. Appl. Phys. 43, 7109-7110 (2004).

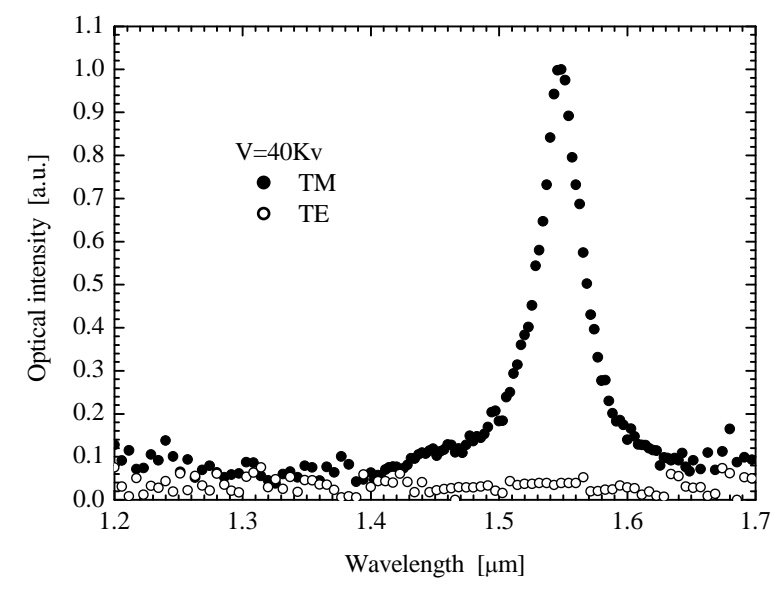

Fig.3 Difference on electric polarization for emitted light

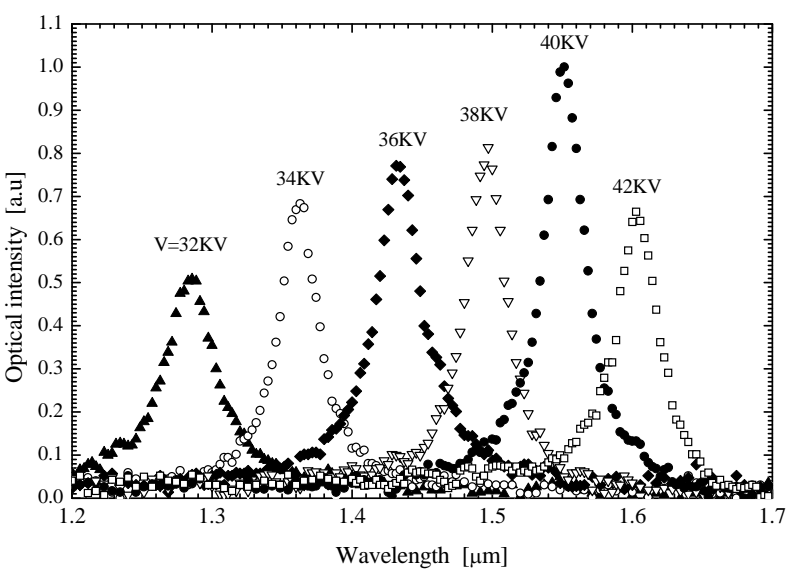

Fig.4 Emission spectrum for different acceleration voltage 